\title{
Existence of a Phase-Transition in a One-Dimensional Ising Ferromagnet
}

\author{
Freeman J. Dyson \\ Institute for Advanced Study, Princeton, New Jersey \\ Received October 28, 1968
}

\begin{abstract}
Existence of a phase-transition is proved for an infinite linear chain of spins $\mu_{j}= \pm 1$, with an interaction energy

$$
H=-\sum J(i-j) \mu_{i} \mu_{j},
$$

where $J(n)$ is positive and monotone decreasing, and the sums $\Sigma J(n)$ and $\sum(\log \log n)\left[n^{3} J(n)\right]^{-1}$ both converge. In particular, as conjectured by KAC and Thompson, a transition exists for $J(n)=n^{-\alpha}$ when $1<\alpha<2$. A possible extension of these results to Heisenberg ferromagnets is discussed.
\end{abstract}

\section{Introduction}

We consider the one-dimensional Ising ferromagnet with sites labeled by an integer $j$ taking all values from $-\infty$ to $+\infty$. At each site is a random variable $\mu_{j}$ taking the values \pm 1 , the total energy being

$$
H=-\sum_{i>j} J(i-j) \mu_{i} \mu_{j}
$$

with

$$
J(n) \geqq 0, \quad n=1,2,3, \ldots
$$

Gallavotit and Mrracle-Sole [1] have proved that this system exists as a well-defined limit of a finite system, allowing a consistent definition of thermodynamic averages, provided that

$$
M_{0}=\sum_{n=1}^{\infty} J(n)
$$

is finite. Since we are assuming (1.2), the case in which $M_{0}$ is infinite is mathematically uninteresting. When $M_{0}$ is infinite there is an infinite energy-gap between the ground states and all other states, so that the system is completely ordered at all finite temperatures, and there can be no question of a phase-transition.

On the other hand, the case in which only a finite number of $J(n)$ are nonzero has long been known [2] to be uninteresting for the opposite reason; the system can have no phase-transition because it is disordered at all finite temperatures. An interesting one-dimensional model considered by BAUR and NosANow [3], giving rise to a phase-transition 7 Commun, math. Phys., Vol. 12 
with only nearest-neighbour interactions, lies outside the scope of this statement since it has some of the interaction constants equal to minus infinity. Recently RUELLE [4] has proved that for any system satisfying (1.2) there is disorder at all finite temperatures, and therefore no phasetransition, provided that

$$
M_{1}=\sum_{n=1}^{\infty} n J(n)
$$

is finite. Intuitively, it seems reasonable that the first moment $M_{1}$ should determine the existence or nonexistence of a phase-transition, because $2 M_{1}$ is the energy required to pass from a perfectly ordered state (all $\left.\mu_{j}=+1\right)$ to a state with a single break in the ordering $\left(\mu_{j}=+1\right.$ for $j \geqq 0, \mu_{j}=-1$ for $j<0$ ). If $M_{1}$ is finite, breaks in the ordering must appear with finite density along the chain at any finite temperature, and so Ruelle's conclusion that no long-range order can exist is to be expected. The converse staiement, that for $M_{1}$ infinite there will be long-range order at sufficiently low temperatures, is plausible but has not been proved. KAC and THompson [5] have studied some particular examples from which they were led to make the

Conjecture (Kac-Thompson). Any system (1.1) satisfying (1.2) with $M_{0}$ finite and $M_{1}$ infinite has a phase-transition at some finite temperature. If this conjecture is true, the occurrence of a phase-transition is completely determined by the two moments $M_{0}$ and $M_{1}$. In particular, the conjecture has the

Corollary (Kac-Thompson). The system (1.1) with

$$
J(n)=n^{-\alpha}
$$

has a phase-transition if and only if

$$
1<\alpha \leqq 2 \text {. }
$$

The present paper arose from an attempt to prove the Kac-Thompson conjecture. We did not succeed in relating our results to the divergence of $M_{1}$. Instead we were led to consider the inverse moment

$$
K_{3}^{\prime}=\sum_{n=1}^{\infty}(\log \log (n+4))\left[n^{3} J(n)\right]^{-1} \text {. }
$$

The 4 is of course only put in to keep all the terms positive. Our main result is then

Theorem 1. In the system (1.1) with (1.2), there is a phase-transition at finite temperature, provided that $J(n)$ is monotonically decreasing and that both $M_{0}$ and $K_{3}^{\prime}$ are finite.

With this follows the

Corollary 1. The system (1.1) with (1.5) has a phase-transition if

$$
1<\alpha<2 \text {. }
$$


The fact that the case $J(n)=n^{-2}$ is left undecided shows that our theorem is weaker than the Kac-Thompson conjecture. We have no definite opinion concerning the occurrence of a transition for $J(n)=n^{-2}$, and we recommend this case as an interesting object for further study [6].

In general, Cauchy's inequality implies

$$
M_{1} \sum f(n)\left[n^{3} J(n)\right]^{-1} \geqq\left[\sum n^{-1}(f(n))^{1 / 2}\right]^{2},
$$

for any positive $f(n)$. Thus we could replace $K_{3}^{\prime}$ in Theorem 1 by either

or

$$
K_{3}=\sum_{n=1}^{\infty}\left[n^{3} J(n)\right]^{-1}
$$

$$
K_{3}^{\prime \prime}=\sum_{n=2}^{\infty}\left[(\log n)^{2} n^{3} J(n)\right]^{-1},
$$

and the resulting statements would still be weaker than the Kac-Thompson conjecture. We consider it likely that the $(\log \log (n+4))$ in $K_{3}^{\prime}$ could be removed by a more careful analysis. Also the condition of monotonicity of $J(n)$ is certainly not essential but is introduced for reasons of technical convenience. One possible alternative to the Kac-Thompson conjecture is the following.

Conjecture (Anti-Kac-Thompson). The necessary and sufficient condition for a phase-transition in the system (1.1) with (1.2) is that both $M_{0}$ and $K_{3}$ be finite.

Neither half of this conjecture is proved. The necessity half is stronger than Ruelle's theorem [4], and the sufficiency half is stronger than our Theorem 1. If the Anti-Kac-Thompson conjecture is true, there is no transition for $J(n)=n^{-2}$.

The proof of Theorem 1 is divided into two unequal parts. The first part (Section 2) is a proof of

Theorem 2 (GRIFFITHS). In the system (1.1) with (1.2) and $M_{0}$ finite, there is zero spontaneous magnetization at all temperatures $T$ such that

$$
k T>2 M_{0} .
$$

This is an immediate consequence of a theorem of GRIFFITHs [7], which states that in any Ising ferromagnet the true spontaneous magnetization does not exceed the value given by a molecular-field approximation. For this half of our theorem, all the hard work has been done by GrIfFIths.

The second half of Theorem 1 may be stated as follows.

Theorem 3. In the system (1.1) with (1.2), if $J(n)$ is monotone decreasing and $K_{3}^{\prime}$ is finite, there is nonzero spontaneous magnetization at all temperatures $T$ such that

$$
\beta=(k T)^{-1}>A K_{3}^{\prime},
$$

with $A$ an absolute constant. 
We shall in fact prove Theorem 3 with $A=2^{10}$. The proof occupies the main part of this paper. It is based on another theorem of GRIFFITHS [8], which states that in any Ising ferromagnet the spontaneous magnetization can only decrease when any interaction-constant is decreased. Theorem 3 will follow if we can construct a simplified Ising ferromagnet which has nonzero spontaneous magnetization and has interaction less than $J(i-j)$ between every pair of sites $i$ and $j$. The simplified system is called the "Hierarchical Model" and is fully described in Section 3. The main virtue of the hierarchical model is that it has a much higher degree of symmetry than the system (1.1). A finite chain of the form (1.1) with $l$ sites arranged in a circle has a symmetry-group of order $2 l$, whereas the hierarchical model with $l$ sites has a symmetry-group of order $2^{l-1}$. We are not able to compute the spontaneous magnetization of the hierarchical model exactly (this is another unsolved problem which we recommend to others to pursue), but in Section 4 we compute lower bounds which remain nonzero in the limit as $l \rightarrow \infty$ provided (1.13) holds.

The final Section 5 is concerned with the possible extension of these results to a Heisenberg ferromagnet, in which there is a quantummechanical spin $s_{j}$ at each site and the scalar product $\left(s_{i} \cdot s_{j}\right)$ replaces $\mu_{i} \mu_{j}$ in the interaction (1.1). For this case neither of the two crucial theorems of GRIfFiths $[7,8]$ is proved. Nevertheless the definition and analysis of the hierarchical model can be carried through, and the existence of a spontaneous magnetization can be proved in the hierarchical Heisenberg model under the same conditions as for the Ising model. It seems extremely likely (and desirable) that the two Griffiths theorems will one day be extended to Heisenberg systems, and it will then follow immediately that our Theorems 1, 2 and 3 will also hold for Heisenberg ferromagnets.

\section{Proof of Theorem 2}

The theorem of GRIFFITHs [7] states that for the system (1.1) with (1.2), for any two distinct sites $i$ and $j$,

$$
\left\langle\mu_{i} \mu_{j}\right\rangle \leqq \tanh (\beta J(i-j))+\sum_{k \neq i, j} \tanh (\beta J(k-j))\left\langle\mu_{i} \mu_{k}\right\rangle,
$$

where the averages are taken for a statistical ensemble at temperature $T=(k \beta)^{-1}$. Griffiths proves (2.1) for a finite system, but the passage to the limit of an infinite chain is justified provided that the sum

$$
U=\sum_{k \neq j} \tanh (\beta J(k-j))<2 \beta M_{0}
$$

is absolutely convergent. The hypothesis of Theorem 2 ensures that $M_{0}$ is finite, and so (2.1) holds. Let (2.1) now be summed over any finite set 
$S$ of sites $i$. The result is

$$
\left(\operatorname{Max}_{j} \sum_{i \varepsilon S, i \neq j}\left\langle\mu_{i} \mu_{j}\right\rangle\right)(1-U) \leqq U
$$

If (1.12) holds, then $U<1$, and therefore

$$
\sum_{i \varepsilon S}\left\langle\mu_{i} \mu_{j}\right\rangle
$$

is bounded uniformly in $S$ and $j$. This means that the infinite sum

$$
\sum_{i \neq j}\left\langle\mu_{i} \mu_{j}\right\rangle, \quad j \text { fixed }
$$

must converge absolutely, and in particular

$$
\left\langle\mu_{i} \mu_{j}\right\rangle \rightarrow 0 \text { as }|i-j| \rightarrow \infty .
$$

Therefore there is no spontaneous magnetization, and Theorem 2 is proved.

\section{The Hierarchical Model}

For each positive integer $N$ a finite hierarchical model $M_{N}$ is defined as follows. There are $2^{N}$ spins $\mu_{j}= \pm 1$, labeled by the index $j=1,2, \ldots, 2^{N}$. For each pair of integers $p=0,1,2, \ldots, N ; r=1,2, \ldots, 2^{N-p}$, we consider the spin-sum

$$
S_{p, r}=\sum \mu_{j}, \quad(r-1) 2^{p}+1 \leqq j \leqq r 2^{p} .
$$

This is the sum of the $r^{\prime}$ th block of $2^{p}$ consecutive spins. The hierarchical character of these sums is expressed by the relation

$$
S_{p, r}=S_{p-1,2 r-1}+S_{p-1,2 r}, \quad p=1,2, \ldots, N .
$$

We assume the interaction energy in the model $M_{N}$ to be

$$
H_{N}=-\sum_{p=1}^{N} 2^{-2 p} b_{p} \sum_{r=1}^{2^{N-p}}\left(S_{p, r}\right)^{2},
$$

where $b_{1}, \ldots, b_{N}$ are non-negative coefficients. The statistical properties of the model are completely defined, given (3.3) and a temperature $T=(k \beta)^{-1}$.

The high degree of symmetry of the model is shown by the fact that every block of spins $S_{p, r}$ is equivalent to every other $S_{p, s}$ with the same $p$. The model has no linear order and no end-effects. In particular, all sites $j$ are equivalent. To each pair of sites $(j, k)$ there corresponds a unique integer $p(j, k)$ such that $\left(\mu_{j}, \mu_{k}\right)$ belong to a common $S_{p, r}$ but not to a common $S_{p-1, r}$. All pairs of sites $(j, k)$ with the same $p(j, k)$ are equivalent, and each pair contributes to $H_{M}$ an interaction

$$
-R_{N}(p) \mu_{j} \mu_{k}, \quad R_{N}(p)=\sum_{q=p}^{N} 2^{1-2 q} b_{q} .
$$


The expectation-value of $\left(\mu_{j} \mu_{k}\right)$ in the model $M_{N}$ will also depend only on $p(j, k), N$ and $\beta$, and so we write

$$
\left\langle\mu_{j} \mu_{k}\right\rangle_{N}=c_{N}(p), \quad p=p(j, k),
$$

the dependence on $\beta$ being understood. When $j=k$ we have $p(j, k)=0$, and so it is consistent with (3.5) to write

$$
c_{N}(0)=1 \text {. }
$$

It follows from the definitions of $p(j, k)$ and $S_{p, r}$ that

where

$$
c_{N}(p)=2 f_{N}(p)-f_{N}(p-1), \quad p=1, \ldots, N,
$$

$$
f_{N}(p)=2^{-2 p}\left\langle\left(S_{p, r}\right)^{2}\right\rangle_{N}, \quad f_{N}(0)=1,
$$

and $f_{N}(p)$ is independent of $r$ by symmetry. It follows from Griffitus' theorems [8] that both $f_{N}(p)$ and $c_{N}(p)$ are positive and increasing functions of $N$ for fixed $p$,

$$
\begin{aligned}
& 0 \leqq c_{N}(p) \leqq c_{N+1}(p) \leqq 1 \\
& 0 \leqq f_{N}(p) \leqq f_{N+1}(p) \leqq 1 .
\end{aligned}
$$

We shall prove that they are also decreasing functions of $p$ for fixed $N$,

$$
\begin{aligned}
& c_{N}(p-1) \geqq c_{N}(p), \\
& f_{N}(p-1) \geqq f_{N}(p) .
\end{aligned}
$$

In fact, (3.11) is a trivial consequence of (3.2) and (3.7). To prove (3.10), let $(x, y, z, w)$ denote four consecutive spin-sums $S_{p-2, r}$, so that $(x+y$, $z+w)$ are sums $S_{p-1, r}$, and $(x+y+z+w)$ is a sum $S_{p, r}$. Then (3.10) is equivalent to the statement

$$
\begin{gathered}
\left\langle(x+y)^{2}+(z+w)^{2}\right\rangle_{N} \geqq \frac{1}{3}\left\langle(x+y)^{2}+(z+w)^{2}+(x+z)^{2}\right. \\
\left.+(y+w)^{2}+(x+w)^{2}+(y+z)^{2}\right\rangle_{N} .
\end{gathered}
$$

But the statistical weighting factor $\exp \left(-\beta H_{N}\right)$ is a symmetric function of $(x, y, z, w)$ multiplied by the factor

$$
\phi=\exp \left(\beta 2^{2-2 p} b_{p-1}\left((x+y)^{2}+(z+w)^{2}\right)\right) .
$$

Thus (3.12) follows from a simple case of Chebychev's inequality [9], and (3.10) is proved.

The sum of the interaction coefficients (3.4) linking a given spin $\mu_{j}$ with all others is

$$
R_{N}=\sum_{p=1}^{N} 2^{p-1} R_{N}(p)=\sum_{q=1}^{N}\left(2^{q}-1\right) 2^{1-2 q} b_{q} .
$$

If the sum

$$
R=\sum_{q=1}^{\infty}\left(2^{q}-1\right) 2^{1-2 q} b_{q}
$$


converges, we can define an infinite hierarchical model by letting $N \rightarrow \infty$ in (3.3) and applying the argument of Gallavotrs and Miracle-Sole [1]. The correlation-functions (3.5) and (3.7) will, by virtue of (3.8) and (3.9), tend to limits

$$
c_{N}(p) \rightarrow c(p), \quad f_{N}(p) \rightarrow f(p), \quad N \rightarrow \infty,
$$

which are the correlation-functions of the infinite model. In view of (3.6), (3.10) and (3.11),

$$
\begin{aligned}
c(p) & =2 f(p)-f(p-1), \\
c(p-1) & \geqq c(p), \quad f(p-1) \geqq f(p) .
\end{aligned}
$$

The spontaneous magnetization of the infinite model is the quantity $m$ defined by

$$
m^{2}=\lim _{p \rightarrow \infty} f(p)=\lim _{p \rightarrow \infty} c(p), \quad 0 \leqq m \leqq 1 .
$$

The two limits in (3.19) must exist and be equal by virtue of (3.17) and (3.18). Moreover

$$
f(p) \geqq c(p) \geqq m^{2}
$$

for every finite $p$. Our main objective will be to find conditions on the coefficients $b_{p}$ and the temperature $T$, to decide whether the spontaneous magnetization of the infinite hierarchical model is or is not zero. In the high-temperature domain we can prove, by a word-for-word repetition of the proof of Theorem 2, again using Griffiths' theorem [8],

Theorem 4. The infinite hierarchical model has zero spontaneous magnetization at all temperatures satisfying

when $R$ given by (3.15) is finite.

$$
\beta R<1,
$$

In the low-temperature domain we shall prove

Theorem 5. If the sum

$$
L=\sum_{q=1}^{\infty}(\log (1+q))\left(b_{q}\right)^{-1}
$$

converges, the infinite hierarchical model has nonzero spontaneous magnetization for all temperatures satisfying

$$
\beta>8 L \text {. }
$$

The proof of Theorem 5 is postponed to Section 4 since it requires some detailed analysis. Theorems 4 and 5 together show that the hierarchical model exhibits a phase transition at a finite temperature if both the sums $R$ and $L$ are finite. In particular, there is a transition if $b_{p}=2^{(2-\alpha) p}$ and $1<\alpha<2$. In the opposite direction we have an analog of Ruelle's theorem [4], namely

Theorem 6. The infinite hierarchical model has zero spontaneous magnetization at all temperatures if the $b_{p}$ are bounded. 
Theorems 4, 5 and 6 together imply the

Corollary 2. The infinite hierarchical model with

$$
b_{p}=2^{(2-\alpha) p}
$$

has a phase-transition at a finite temperature if and only if

$$
1<\alpha<2 .
$$

Roughly speaking, we expect the linear model with $J(n)=n^{-\alpha}$ to behave like the hierarchical model with (3.24). The average strength of the interaction between two spins at a large distance is the same in both models. Corollaries 1 and 2 show that both models have phase-transitions in the same range $1<\alpha<2$. However, the critical case $\alpha=2$, for which the question of a transition is left open for the linear model by Theorem 1 and the Ruelle theorem, is settled for the hierarchical model by Theorem 6 . The fact that the hierarchical model has no transition for $\alpha=2$ provides support, but no proof, for the opinion [6] that the same is true for the linear model.

We conclude this section with a proof of Theorem 6. The proof is short, unlike the proof of Ruelle's theorem [4], and makes essential use of the symmetry of the hierarchical model. The only tool required for the proof is Griffiths' theorem [8], which is used three times.

First use of Griffiths. If $b_{p} \leqq b_{p}^{\prime}$ and if the hierarchical model with coefficients $b_{p}^{\prime}$ has zero spontaneous magnetization, then the same is true for the $b_{p}$. Therefore Theorem 6 holds in general if we can prove it for $b_{p}$ independent of $p$. The theorem holds for $b_{p}=M$ at temperature $T$ if it holds for $b_{p}=1$ at temperature $(T / M)$. Therefore it is sufficient to prove Theorem 6 for

$$
b_{p}=1 \text {, }
$$

which is just the critical case $\alpha=2$ of (3.24).

Second use of Griffiths. Let $\left(\mu, \mu^{\prime}\right)$ be any pair of neighbouring spins forming a sum $S_{1, r}$ according to (3.1) in a finite hierarchical model $M_{N}$. Let $p_{N}=f_{N}(1)$ be the probability that $\left(\mu, \mu^{\prime}\right)$ are parallel in $M_{N}$. By Griffiths' theorem [8] the value of $p_{N}$ does not decrease if all the spins except $\mu$ are locked in a parallel orientation by infinite attractive interactions. Thus by (3.14)

$$
\begin{aligned}
p_{N} & \leqq\left[1+\exp \left(-2 \beta R_{N}\right)\right]^{-1}<[1+\exp (-2 \beta R)]^{-1} \\
& =[1+\exp (-8 \beta / 3)]^{-1}=g<1 .
\end{aligned}
$$

Third use of Griffiths. For any integer $q$ with $1 \leqq q \leqq N$, we have by $(3.7)$

$$
f_{N}(q)=2^{-1-q}\left\langle\left(\mu+\mu^{\prime}\right) S_{q, r}\right\rangle_{N},
$$

where $S_{q, r}$ is the particular block of $2^{q}$ spins in which $\mu$ and $\mu^{\prime}$ lie. By the definition of $p_{N},(3.28)$ implies

$$
f_{N}(q)=2^{-q} p_{N}\left\langle\mu S_{q, r}\right\rangle_{N, L},
$$


where the average is taken over the model $M_{N}$ with the two spins $\left(\mu, \mu^{\prime}\right)$ locked parallel. Now the Griffiths theorem [8] states that the average in (3.29) will not decrease if every pair of neighbour spins in $M_{N}$ is locked parallel. But the model $M_{N}$ with every pair of neighbour spins locked, and with the particular set of coefficients (3.26), is identical to the model $M_{N-1}$ with the same coefficients. At this point the symmetry of the hierarchical model plays an essential role. Thus Griffiths' theorem applied to $(3.29)$ gives

$$
f_{N}(q) \leqq 2^{1-q} p_{N}\left\langle\mu S_{q-1, r}\right\rangle_{N-1}=p_{N} f_{N-1}(q-1),
$$

and so by $(3.27)$ and $(3.7)$

$$
f_{N}(q)<g f_{N-1}(q-1)<g^{q} f_{N-q}(0)=g^{q} .
$$

Going to the limit $N \rightarrow \infty$ by (3.16), we have for the infinite hierarchical model with (3.26),

and so (3.19) gives

$$
f(q) \leqq g^{q}, \quad g<1
$$

$$
m^{2}=0,
$$

which is the conclusion of Theorem 6 .

\section{Existence of Long-Range Order}

We now come to the most difficult part of this investigation, the proof of existence of long-range order for the linear model (Theorem 3) and for the hierarchical model (Theorem 5). We first deduce Theorem 3 from Theorem 5 and then prove Theorem 5.

Suppose then that Theorem 5 is true and that the hypotheses of Theorem 3 are satisfied. We have a linear Ising ferromagnet with energy (1.1) satisfying (1.2), the coefficients $J(n)$ decreasing monotonically and the temperature satisfying (1.13). We shall relate this linear model to the hierarchical model (3.3) with coefficients defined by

$$
b_{p}=\operatorname{Min}_{1 \leqq q \leqq p} 2^{p+q-2} J\left(2^{q}-1\right) .
$$

Any block of $2^{N}$ sites in the linear model can be identified with the sites of a finite hierarchical model $M_{N}$. The interaction between a pair of sites $(j, k)$ in $M_{N}$ is then given by (3.4) with

and is

$$
p=p(j, k), \quad|j-k| \leqq 2^{p}-1,
$$

$$
\begin{aligned}
R_{N}(p) & =\sum_{q=p}^{N} 2^{1-2 q} \underset{1 \leqq r \leqq q}{\operatorname{Min}} 2^{q+r-2} J\left(2^{r}-1\right) \\
& \leqq \sum_{q=p}^{N} 2^{p-q-1} J\left(2^{p}-1\right)<J(|j-k|),
\end{aligned}
$$


the last inequality by virtue of the monotonicity of $J(n)$. The meaning of (4.3) is that our choice of coefficients (4.1) gives a model $M_{N}$ with all interactions weaker than those of the linear model. The Griffiths theorem [8] then states that

$$
\left\langle\mu_{j} \mu_{k}\right\rangle_{L} \geqq\left\langle\mu_{j} \mu_{k}\right\rangle_{N}=c_{N}(p),
$$

where $L$ denotes an average in the linear model, for every pair of sites $(j, k)$ and every integer $N \geqq p$. Going to the limit $N \rightarrow \infty$ by (3.16) and using (3.20), we deduce

$$
\left\langle\mu_{j} \mu_{k}\right\rangle_{L} \geqq c(p) \geqq m^{2},
$$

where $m$ is the spontaneous magnetization of the infinite hierarchical model. The linear model therefore has nonzero spontaneous magnetization and Theorem 3 holds, if it can be shown that (1.13) implies $m \neq 0$.

Some crude estimating applied to (1.13), using the monotonicity of $J(n)$, gives

$$
\begin{aligned}
\beta & >A \sum_{r=1}^{\infty} \sum_{2^{r-1} \leqq n \leqq 2^{r+1}-2}\left(n^{-3} \log \log (n+4)\right)\left[J\left(2^{r}-1\right)\right]^{-1} \\
& >A \sum_{r=1}^{\infty}\left(\log \log \left(2^{r}+3\right)\right) 2^{-2-2 r}\left[J\left(2^{r}-1\right)\right]^{-1} \\
& >A \sum_{r=1}^{\infty}(\log (1+r)) 2^{-3-2 r}\left[J\left(2^{r}-1\right)\right]^{-1}
\end{aligned}
$$

On the other hand, Theorem 5 with (4.1) states that $m \neq 0$ provided that

$$
\beta>8 \sum_{q=1}^{\infty} \log (1+q) \underset{1 \leqq r \leqq q}{\operatorname{Max}} 2^{2-q-r}\left[J\left(2^{r}-1\right)\right]^{-1} .
$$

This inequality is strengthened if we replace the Max by a summation over $r$. After this change and a little more algebra, (4.7) gives as a sufficient condition for $m \neq 0$

$$
\beta>2^{7} \sum_{r=1}^{\infty} \log (1+r) 2^{-2 r}\left[J\left(2^{r}-1\right)\right]^{-1} .
$$

Therefore Theorem 3 follows from Theorem 5 provided that

$$
A \geqq 2^{10} \text {. }
$$

We have not made any attempt to reduce the size of $A$ by more careful numerical estimates.

At this point we begin the proof of Theorem 5. We assume a set of positive coefficients $b_{p}$, no longer defined by (4.1), but satisfying only (3.22) and (3.23). The hierarchical model $M_{N}$ is constructed with these coefficients. The partition function of $M_{N}$ is a sum of terms

$$
Z_{N}=\sum_{s} Z_{N}(s)
$$


where $s$ denotes the sum of all the $\mu_{j}$ and takes values from $-2^{N}$ to $+2^{N}$ in steps of 2 . Each $Z_{N}(s)$ is a sum over the configurations of $M_{N}$ with given $s$. All our analysis is done at a fixed temperature, and so the dependence of these quantities on $\beta$ is not written explicitly.

It is convenient to introduce a new real parameter $u$ and to define

$$
\begin{aligned}
& \zeta_{N}(u)=\sum_{s} \exp \left(u s^{2}\right) Z_{N}(s), \\
& \zeta_{N}(0)=Z_{N} .
\end{aligned}
$$

The degree of order of the model is then measured by the quantity

$$
f_{N}(N)=2^{-2 N}\left\langle s^{2}\right\rangle_{N}=2^{-2 N} L_{N}^{\prime}(0),
$$

according to $(3.7)$, where

$$
L_{N}(u)=\log \zeta_{N}(u) .
$$

We shall make essential use of the fact that, by (4.11), $L_{N}(u)$ is a convex function of $u$.

The hierarchical character of the energy (3.3) leads to a recurrence relation between the $Z_{N}(s)$, namely

with

$$
\zeta_{N}(u)=\sum_{x} \sum_{y} \exp \left((u+v)(x+y)^{2}\right) Z_{N-1}(x) Z_{N-1}(y),
$$

$$
v=2^{-2 N} \beta b_{N} .
$$

Here both variables $x$ and $y$ take values from $-2^{N-1}$ to $+2^{N-1}$ in steps of 2 . In particular, taking $u=-v$ in (4.15)

$$
\zeta_{N}(-v)=\left[\zeta_{N-1}(0)\right]^{2} \text {. }
$$

When $u=0$ we estimate the right side of (4.15) by means of

Lemma 1. Let $x_{j}$ be a discrete variable taking values in the range

$$
0 \leqq x_{j} \leqq a,
$$

let $f(x)$ be a positive real function and $b$ a real number. Then

$$
\sum_{j} \sum_{k} \exp \left[-\left(x_{j}-x_{k}\right)^{2} / b^{2}\right] f\left(x_{j}\right) f\left(x_{k}\right) \geqq e^{-1}(b /(a+b))\left[\sum_{j} f\left(x_{j}\right)\right]^{2} .
$$

The proof of Lemma 1 is elementary and is given at the end of this section. We apply the lemma to (4.15) with

$$
\begin{gathered}
x_{j}=x+2^{N-1}, \quad x_{k}=y+2^{N-1}, \quad a=2^{N}, \\
b=v^{-1 / 2}=2^{N}\left(\beta b_{N}\right)^{-1 / 2}, \\
f\left(x_{j}\right)=\exp \left(2 v x^{2}\right) Z_{N-1}(x) .
\end{gathered}
$$

The conclusion (4.19) then becomes

$$
\zeta_{N}(0) \geqq e^{-1}\left[1+\left(\beta b_{N}\right)^{1 / 2}\right]^{-1}\left[\zeta_{N-1}(2 v)\right]^{2} .
$$


Taking logarithms of (4.21) and (4.17) and subtracting, we find

$$
\begin{aligned}
L_{N}(0) & -L_{N}(-v) \geqq 2\left[L_{N-1}(2 v)-L_{N-1}(0)\right] \\
& -1-\log \left[1+\left(\beta b_{N}\right)^{1 / 2}\right] .
\end{aligned}
$$

Now the convexity of $L_{N}(u)$ and $L_{N-1}(u)$ implies

$$
\begin{aligned}
L_{N}(0)-L_{N}(-v) & \leqq v L_{N}^{\prime}(0), \\
L_{N-1}(2 v)-L_{N-1}(0) & \geqq 2 v L_{N-1}^{\prime}(0) .
\end{aligned}
$$

Therefore (4.13), (4.16) and (4.22) together give

$$
\begin{aligned}
f_{N}(N) & \geqq 2^{-2 N}\left[2^{2 N} f_{N-1}(N-1)-v^{-1}\left(1+\log \left(1+\left(\beta b_{N}\right)^{1 / 2}\right)\right)\right] \\
& =f_{N-1}(N-1)-\left(\beta b_{N}\right)^{-1}\left(1+\log \left(1+\left(\beta b_{N}\right)^{1 / 2}\right)\right) .
\end{aligned}
$$

Since $f_{0}(0)=1$, the repeated use of $(4.25)$ gives

$$
f_{N}(N) \geqq 1-\sum_{p=1}^{N}\left(\beta b_{p}\right)^{-1}\left(1+\log \left(1+\left(\beta b_{p}\right)^{1 / 2}\right)\right) .
$$

To simplify (4.26), we divide the sum into two parts according as $\beta b_{p}$ is greater than or less than $9 p^{2}$. The terms with $\beta b_{p}>9 p^{2}$ cannot exceed

$$
\sum_{p=1}^{\infty}\left(9 p^{2}\right)^{-1}(1+\log (1+3 p))<9 / 16
$$

The remaining terms cannot exceed

$$
\sum_{p=1}^{\infty}\left(\beta b_{p}\right)^{-1}(1+\log (1+3 p))<(7 / 2) \beta^{-1} L,
$$

where $L$ is defined by (3.22) and is assumed to be finite. Thus (4.26) implies

$$
f_{N}(N) \geqq(7 / 16)\left[1-8 \beta^{-1} L\right] .
$$

Passing now to the infinite hierarchical model, we have by (3.9) and (3.16)

$$
f(p)=\lim _{N \rightarrow \infty} f_{N}(p) \geqq f_{p}(p),
$$

and finally (3.19) and (4.29) give

$$
m^{2} \geqq(7 / 16)\left[1-8 \beta^{-1} L\right] .
$$

When (3.23) holds, the spontaneous magnetization is nonzero, and so Theorem 5 is proved.

It remains only to prove Lemma 1. Let $q$ be the integer defined by

$$
(a / b)<q \leqq 1+(a / b),
$$

and divide the interval (4.18) into $q$ equal parts $I_{p}, p=1,2, \ldots, q$. Let $F_{p}$ be the sum of the $f\left(x_{j}\right)$ with $x_{j}$ in $I_{p}$. If we drop from the left side of 
(4.19) all terms with $\left(x_{j}, x_{k}\right)$ in different $I_{p}$, we obtain

$$
\begin{aligned}
\sum_{j} & \sum_{k} \exp \left[-\left(x_{j}-x_{k}\right)^{2} / b^{2}\right] f\left(x_{j}\right) f\left(x_{k}\right) \\
& \geqq \exp \left[-a^{2} / q^{2} b^{2}\right] \sum_{p}\left(F_{p}\right)^{2} \\
& >e^{-1} q^{-1}\left(\sum F_{p}\right)^{2},
\end{aligned}
$$

from which (4.19) follows by virtue of (4.32). The proofs of Theorems 5,3 and 1 are thereby completed.

\section{Heisenberg Ferromagnets}

Consider a finite hierarchical model $K_{N}$ of a Heisenberg ferromagnet, defined by the interaction energy (3.3), where now $S_{p, r}$ is a vector

$$
S_{p, r}=\sum s_{j}, \quad(r-1) 2^{p}+1 \leqq j \leqq r 2^{p},
$$

and each $s_{j}$ is a vector representing a quantum-mechanical spin at the site $j$. The individual $s_{j}$ represent the angular momentum of a particle with spin $1 / 2$, so that

$$
s_{j}^{2}=3 / 4, \quad j=1, \ldots, 2^{N} .
$$

The states of the model $K_{N}$ form a finite vector-space of dimension $2^{2^{N}}$, in which the operators $S_{p, r}$ are defined.

It is easy to verify by induction on $N$ the following statements. (1) The operators $\left(S_{p, r}\right)^{2}$ all commute with one another and with the total spin $S_{N, 1}$. (2) A complete set of commuting operators consists of

$$
\left(S_{p, r}\right)^{2}, \quad p=1,2, \ldots, N ; \quad r=1,2, \ldots, 2^{N-p},
$$

and one component $S_{N, 1, z}$ of $S_{N, 1}$. (3) There is one and only one state of $K_{N}$ having the eigenvalues

$$
\begin{gathered}
j_{p, r}\left(j_{p, r}+1\right) \text { for }\left(S_{p, r}\right)^{2}, \\
m \text { for } S_{N, \mathbf{1}, z},
\end{gathered}
$$

where $j_{p, r}$ and $m$ are integers satisfying the inequalities

$$
\begin{aligned}
0 \leqq j_{1, r} \leqq 1, \quad r & =1,2, \ldots, 2^{N-1} \\
\left|j_{p-1,2 r-1}-j_{p-1}, 2 r\right| \leqq j_{p, r} & \leqq j_{p-1,2 r-1}+j_{p-1,2 r} \\
p=2,3, \ldots, N ; \quad r & =1,2, \ldots, 2^{N-p} \\
-j_{N, 1} \leqq m & \leqq+j_{N, 1} .
\end{aligned}
$$

(4) The Hamiltonian $H_{N}$ is diagonal in the representation with the basic states labeled by the quantum-numbers $j_{p, r}$ and $m$, and its eigenvalues are

$$
H_{N}\left(j_{p, r}\right)=-\sum_{p=1}^{N} 2^{-2 p} b_{p} \sum_{r=1}^{2 N-p} j_{p, r}\left(j_{p, r}+1\right) .
$$


In consequence of these statements, the partition-function $Z_{N}$ of the Heisenberg hierarchical model can be handled as if it were a classical system, by-passing all the quantum-mechanical difficulties that make the linear Heisenberg model intractable. We write $Z_{N}$ as a sum of terms (4.10), where now $s$ denotes $j_{N, 1}$ and takes all integer values from 0 to $2^{N-1}$. Instead of (4.11) we write

$$
\zeta_{N}(u)=\sum_{s} \exp (u s(s+1)) Z_{N}(s),
$$

and the recurrence relation $(4.15)$ is replaced by

$$
\begin{aligned}
\zeta_{N}(u)=\sum_{x} \sum_{y} Z_{N-1}(x) Z_{N-1}(y) \sum_{s=|x-y|}^{x+y} \frac{(2 s+1)}{(2 x+1)(2 y+1)} \\
\exp ((u+v) s(s+1)) .
\end{aligned}
$$

As before, (5.10) and (5.11) give

$$
\begin{aligned}
\zeta_{N}(0) & =Z_{N}, \\
\zeta_{N}(-v) & =\left[\zeta_{N-1}(0)\right]^{2},
\end{aligned}
$$

with $v$ defined by (4.16).

We define the coefficients

as in (3.5), and

$$
c_{N}(p)=\left\langle s_{j} \cdot s_{k}\right\rangle_{N}, \quad p=p(j, k),
$$

as in (3.7), only now

$$
f_{N}(p)=2^{-2 p}\left\langle\left(S_{p, r}\right)^{2}\right\rangle_{N}
$$

$$
c_{N}(0)=f_{N}(0)=3 / 4 \text {. }
$$

The monotonicity relations (3.10) and (3.11) still hold, but (3.8) and (3.9) depend on the Griffiths' theorem [8] and cannot be taken as proved for Heisenberg models. The order-parameter $f_{N}(N)$ is again given by (4.13) and (4.14), and $L_{N}(u)$ is still a convex function of $u$.

We can carry through the estimation of the right side of (5.11) in complete analogy with (4.15). Using the elementary bounds

we find

$$
b>a^{-1}(1-\exp (-a b))>b(1+a b)^{-1},
$$

$$
\begin{gathered}
\sum_{s=|x-y|}^{x+y}(2 s+1) \exp (v s(s+1)) \\
>\sum_{s} v^{-1}\left(\exp \left(v(s+1)^{2}\right)-\exp \left(v s^{2}\right)\right) \exp (-v(x+y+1)) \\
=v^{-1} \exp (v(x+y)(x+y+1)) \\
\cdot(1-\exp (-v(2 x+1)(2 y+1))) \\
>\exp (v(x+y)(x+y+1))(2 x+1)(2 y+1) \\
\cdot[1+v(2 x+1)(2 y+1)]^{-1} .
\end{gathered}
$$


Now

and so by (4.16)

$$
x \leqq 2^{N-2}, \quad y \leqq 2^{N-2},
$$

$$
\begin{aligned}
v(x+y)(x+y+1) & >2 v\left(x^{2}+x+y^{2}+y\right) \\
& -v(x-y)^{2}-2^{-1-N} \beta b_{N} .
\end{aligned}
$$

Thus (5.11) and (5.18) give

$$
\begin{aligned}
\zeta_{N}(0) \geqq & \sum_{x} \sum_{y} Z_{N-1}(x) Z_{N-1}(y)\left[1+\beta b_{N}\right]^{-1} \\
& \cdot \exp \left[2 v\left(x^{2}+x+y^{2}+y\right)-v(x-y)^{2}-2^{-1-N} \beta b_{N}\right],
\end{aligned}
$$

and Lemma 1 can again be used. The result of using Lemma 1 is

$$
\begin{gathered}
\zeta_{N}(0) \geqq e^{-1}\left[1+\frac{1}{2}\left(\beta b_{N}\right)^{1 / 2}\right]^{-1}\left[1+\beta b_{N}\right]^{-1} \\
\cdot \exp \left[-2^{-1-N} \beta b_{N}\right]\left[\zeta_{N-1}(2 v)\right]^{2}
\end{gathered}
$$

Putting together (5.23) with (5.13), we find instead of (4.22)

$$
\begin{aligned}
L_{N}(0) & -L_{N}(-v) \geqq 2\left[L_{N-1}(2 v)-L_{N-1}(0)\right] \\
& -1-3 \log \left[1+\left(\beta b_{N}\right)^{1 / 2}\right]-2^{-1-N} \beta b_{N} .
\end{aligned}
$$

Using the convexity of $L_{N}(u)$ as before, this inequality implies

$$
\begin{aligned}
f_{N}(N) & \geqq f_{N-1}(N-1) \\
& -\left(\beta b_{N}\right)^{-1}\left(1+3 \log \left(1+\left(\beta b_{N}\right)^{1 / 2}\right)\right)-2^{-1-N} .
\end{aligned}
$$

Repeated use of (5.25) together with (5.16) gives

$$
f_{N}(N) \geqq \frac{1}{4}-\sum_{p=1}^{N}\left(\beta b_{p}\right)^{-1}\left(1+3 \log \left(1+\left(\beta b_{p}\right)^{1 / 2}\right)\right) .
$$

The same argument which led to (4.29) now gives

$$
f_{N}(N) \geqq(1 / 16)-16 \beta^{-1} L .
$$

We are not able to define the spontaneous magnetization of the infinite Heisenberg hierarchical model by (3.19), since we have not proved the monotonicity property (3.9) upon which the existence of the limit in (3.16) depends. Until the existence of limits is proved, it is reasonable to define the spontaneous magnetization by

$$
m^{2}=\lim _{N \rightarrow \infty} f_{N}(N) \text {. }
$$

The statement that $m^{2} \neq 0$ then implies that spins are correlated at arbitrarily large distances, and $m^{2}$ is a quantitative measure of the degree of long-range order. We may then conclude from (5.27) the following. 
Theorem 7. Nonzero long-range order as defined by (5.28) exists in the infinite Heisenberg hierarchical model with energy (3.3), provided that the sum $L$ defined by (3.22) converges and the temperature satisfies

$$
\beta>2^{8} L \text {. }
$$

It is satisfactory that this analog of Theorem 5 can be pushed through for the Heisenberg ferromagnet. It is unsatisfactory that the easier Theorems 2, 4, 6, and the derivation of Theorems 1 and 3 from 5, all depend on the theorems of GrIfFiths $[7,8]$ and cannot yet be extended to Heisenberg systems. Nevertheless there is every reason to believe that Griffiths' theorems are true for Heisenberg ferromagnets. Thus Theorem 7 makes it extremely probable that all the Theorems $1-6$ in fact hold for Heisenberg systems with only some numerical coefficients changed. Since we expect a Heisenberg ferromagnet to have less longrange order than an Ising ferromagnet with the same structure, it would be natural to expect Theorem 5 to fail in the Heisenberg case. Surprisingly, the theorem which seemed most likely to fail turned out to be the only one we could prove.

In view of Theorem 7, we make the definite conjecture that an infinite linear Heisenberg ferromagnet with the energy

$$
H=-\sum_{i>j} J(i-j)\left(s_{i} \cdot s_{j}\right)
$$

will have a phase-transition at a finite temperature, provided that $J(n)$ is positive and monotonically decreasing and that the sums (1.3) and (1.7) converge.

Acknowledgements. I am deeply indebted to Dr. CoLIn J. Thompson for bringing this problem to my attention and for discussing with me the unpublished work which he and Dr. Mark KaC had done on it. I am also indebted to the Battelle Memorial Institute for hospitality at Seattle during their 1968 Rencontre at which this work was begun.

\section{Addendum}

A theorem as good for our purposes as Theorem 2 was proved earlier by Gallavotti, Miracle-Sole and Robinson [10]. They proved Theorem 2 with 5 replacing 2 on the right side of (1.12). They proved in fact the much stronger statement that the thermodynamic averages are analytic functions of $T$ and of the $J(n)$ in the range

$$
k T>5 M_{0} \text {. }
$$

For this reference I am indebted to Dr. RobInson. 


\title{
References
}

1. Gallavotti, G., and S. Miracle-Sole: Commun. Math. Phys. 5, 317 (1967).

2. Rusirbrooke, G., and H. Ursell: Proc. Cambridge Phil. Soc. 44, 263 (1948).

3. BaUr, M., and L. Nosanow: J. Chem. Phys. 37, 153 (1962).

4. Ruelle, D.: Commun. Math. Phys. 9, 267 (1968).

5. KAC, M., and C. J. Thompson: Critical behavior of several lattice models with long-range interaction. Preprint, Rockefeller University, 1968.

6. Thompson, C. J.: (private communication) now believes that there is no transition for $J(n)=n^{-2}$.

7. Griffiths, R. B.: Commun. Math. Phys. 6, 121 (1967).

8. - J. Math. Phys. 8, 478 (1967). See also Kelly, D. G., and S. Sherman: J. Math. Phys. 9, 466 (1968).

9. Hardy, G. H., J. E. Littlewood, and G. Polya: Inequalities, p. 43. Cambridge Univ. Press 1934.

10. Gallavotti, G., S. Miracle-Sole, and D. W. Robinson: Phys. Letters 25 A, 493 (1967).

\author{
F. J. Dyson \\ Institute for Advanced Study \\ Princeton, N.J. 08540 USA
}

